\title{
A Novel RFID Sensing System Using Enhanced Surface Wave Technology for Battery Exchange Stations
}

\author{
Yeong-Lin Lai, ${ }^{1}$ Li-Chih Chang, ${ }^{1}$ Tai-Hwa Liu, ${ }^{2}$ Yi-Chun Sung, ${ }^{2}$ and Cheng-Lun Yin ${ }^{2}$ \\ ${ }^{1}$ Department of Mechatronics Engineering, National Changhua University of Education, Changhua 50007, Taiwan \\ ${ }^{2}$ EPC Solutions Taiwan Inc., 2F., No. 13 Gongye E. 2nd Road, East District, Hsinchu 30075, Taiwan \\ Correspondence should be addressed to Yeong-Lin Lai; yllai@cc.ncue.edu.tw
}

Received 27 February 2014; Accepted 19 April 2014; Published 30 September 2014

Academic Editor: Her-Terng Yau

Copyright (c) 2014 Yeong-Lin Lai et al. This is an open access article distributed under the Creative Commons Attribution License, which permits unrestricted use, distribution, and reproduction in any medium, provided the original work is properly cited.

\begin{abstract}
This paper presents a novel radio-frequency identification (RFID) sensing system using enhanced surface wave technology for battery exchange stations (BESs) of electric motorcycles. Ultrahigh-frequency (UHF) RFID technology is utilized to automatically track and manage battery and user information without manual operation. The system includes readers, enhanced surface wave leaky cable antennas (ESWLCAs), coupling cable lines (CCLs), and small radiation patches (SRPs). The RFID sensing system overcomes the electromagnetic interference in the metallic environment of a BES cabinet. The developed RFID sensing system can effectively increase the efficiency of BES operation and promote the development of electric vehicles which solve the problem of air pollution as well as protect the environment of the Earth.
\end{abstract}

\section{Introduction}

Air pollution, especially $\mathrm{CO}, \mathrm{HC}$, and $\mathrm{NO}_{x}$, generated by vehicles, such as motorcycles and cars, is a very serious problem in many countries. The quantity per year of polluted air exhausted by vehicles continues to reach record highs. Therefore, the replacement of traditional petroleum vehicles with electric vehicles is becoming a global trend. However, batteries and cost are the most important challenges for electric motorcycles and cars. A rechargeable battery is a low cost solution for electric vehicles. As far as the electric motorcycles are concerned, battery exchange stations (BESs) or rapid-charging batteries are required $[1,2]$.

Battery information is of critical importance for the management of the BES. A barcode attached to a battery is one approach to identify each battery in the BES; however, it consumes manpower and time to gather the battery information.

In recent years, radio-frequency identification (RFID) technology [3-15] has been widely used in different applications because of the contactless, long reading distance and multiread characteristics [16-26]. RFID applications include asset management, health care, logistics, security, and so on [27-38]. During long-distance RFID signal transmission, signal interference might occur and result in erroneous or missed readings. In the metallic environment of a BES, electromagnetic radiation is disturbed in a small space. The traditional antenna of an RFID reader radiates far-field electromagnetic waves and occupies a large area. These features are not suitable for a BES which has a closed and small space.

Leaky cable antennas (LCAs) [39-46] have attracted much attention in regard to communication applications. The conventional LCA is widely used for the mobile communications in subways and tunnels.

In this paper, a novel ultrahigh-frequency (UHF) RFID sensing system integrating a flexible enhanced surface wave leaky cable antenna (ESWLCA) with a coupling cable line (CCL) and a small radiation patch (SRP) is proposed incorporating the enhanced surface wave technology in order to overcome the narrow metallic environment of the BES and to lower the manufacturing cost. The ESWLCA has been successfully implemented in a UHF RFID sensing system for the BESs of electric motorcycles. 


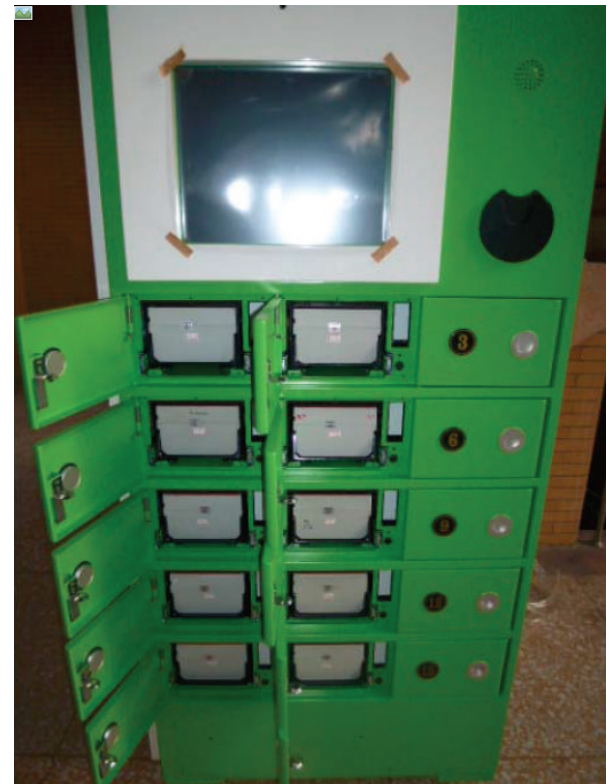

Figure 1: BES.

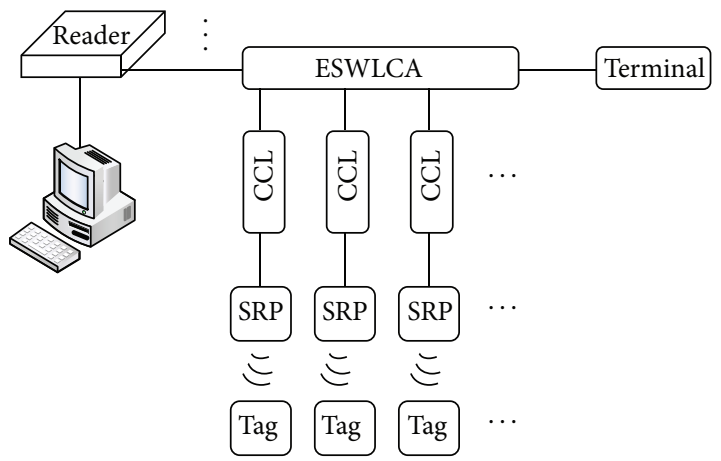

FIGURE 2: System structure of RFID sensing system.

\section{System Structure}

Rechargeable batteries for electric motorcycles are installed in a BES, as shown in Figure 1. A BES is a closed metallic cabinet, in which a large number of dense metal brackets and wires disturb far-field electromagnetic radiation. In addition, the electromagnetic noise reflected by the metallic BES cabinet might cause the saturation and malfunction of the receiver of a reader.

The system structure of the proposed RFID sensing system is shown in Figure 2. The system includes readers, ESWLCAs, CCLs, SRPs, and tags. The ESWLCA transmits the enhanced surface wave through the CCL to the SRP which is close to the tag. The SRP at the end of the CCL radiates the electromagnetic wave to activate the RFID tag attached to the battery. The physical picture of the RFID sensing system with an ESWLCA, a CCL, and an SRP for the BES is shown in Figure 3. The ESWLCA has several advantages, such as flexibility, a slim form factor, and low radiation, to avoid

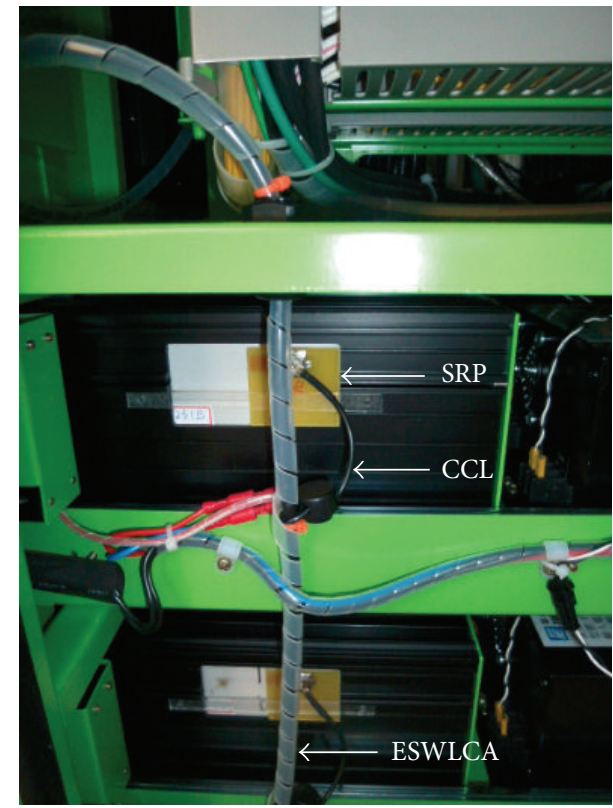

FIGURE 3: RFID sensing system with ESWLCA, CCL, and SRP for BES.

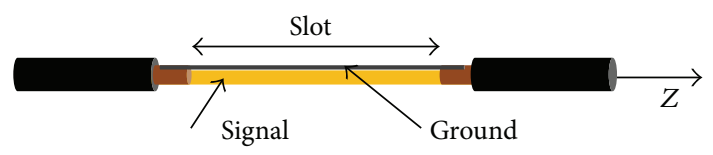

FIGURE 4: Structure of ESWLCA.

interference in the metal-rich environment and to be easily placed in suitable positions for detecting tags. Figure 4 shows the structure of the ESWLCA. There is an open slot on the ESWLCA.

The operation of the system is described as follows. First, one of the ESWLCA ports is connected to the output port of the UHF reader and the other port is terminated with 50 ohms. When the reader turns on the RF power, the RF signal will be fed into the ESWLCA. Some signals will radiate into the air from the slot aperture and some will flow through the cable surface from the slot aperture to produce surface waves along the cable.

Then, at suitable positions of the ESWLCA, the CCL will be connected to the ESWLCA by wiring, so that some surface waves will flow into the CCL from the ESWLCA.

Finally, the surface waves on the CCL will be fed into the port of the radiation patch and the RF energy will be transferred to the RFID tag. As the RF energy is higher than the threshold energy of the RFID tag, communication between the reader and the tag will happen.

\section{System Theorem}

An LCA has the functions of transmission and radiation of electromagnetic waves. When the electromagnetic waves pass the open slot of the LCA, some electromagnetic waves will 


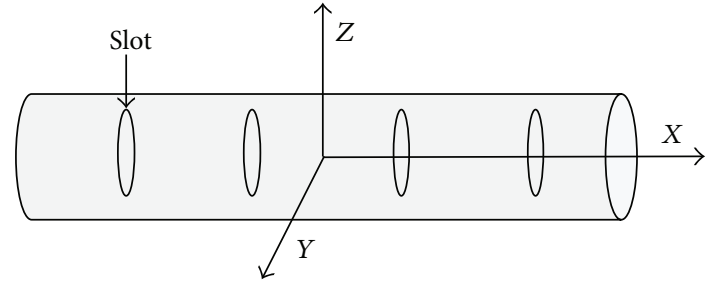

FIGURE 5: Conventional LCA.

leak through the open slot. The LCA has the advantages of wide operation frequency band, large sensing range, easy deployment, and low cost. Figure 5 shows the conventional LCA. The electric field within the area of the radius $r$ of the LCA is determined by

$$
E\left(r, \varphi, x_{d}\right)=M(\eta, r, \varphi) Z\left(x_{d}\right) e^{j \beta x_{d}} e^{-\alpha x_{d}},
$$

where $\varphi$ is the current density, $x_{d}$ is the distance along the $x$-axis, $\eta$ is the magnetic flux density, $\beta$ is the propagation constant of the electromagnetic wave, $\alpha$ is the attenuation constant of the electromagnetic wave, and $M(\eta, r, \varphi)$ represents the integration of electromagnetic field for specific boundaries.

The refection coefficient $\Gamma$, the voltage $U\left(x_{d}\right)$, and the current $I\left(x_{d}\right)$ along the $x$-axis can be represented, respectively, by

$$
\begin{gathered}
\Gamma\left(x_{d}\right)=\frac{Z_{L}-Z_{0}}{Z_{L}+Z_{0}} e^{-j 2 \beta x_{d}}, \\
U\left(x_{d}\right)=U_{i 0} e^{j \beta x_{d}}\left[1+\Gamma\left(x_{d}\right)\right], \\
I\left(x_{d}\right)=\frac{U_{i 0} e^{j \beta x_{d}}}{Z_{0}}\left[1-\Gamma\left(x_{d}\right)\right],
\end{gathered}
$$

where $U_{i 0}$ is the amplitude of the incident wave at $x_{d}=$ 0 , which is the results of the addition of the characteristic impedance $Z_{0}$ and load impedance $Z_{L}$.

The theorems of the impedance, voltage, and current of the ESWLCA follow those of the conventional LCA. The ESWLCA along the $z$-axis does not need to consider the radiation of the electromagnetic waves. The surface electric field of the ESWLCA, $E_{p}(r, \phi, z)$, is a simplified periodic function of $Z$, as follows:

$$
\begin{gathered}
E(r, \phi, z)=E_{p}(r, \phi, z) e^{-j k_{z} z}, \\
-m f_{1}<f<-m f_{2}, \quad m<0, \\
f_{1}=\frac{c}{P\left(\sqrt{\varepsilon_{r}}+1\right)}, \\
f_{2}=\frac{c}{P\left(\sqrt{\varepsilon_{r}}-1\right)},
\end{gathered}
$$

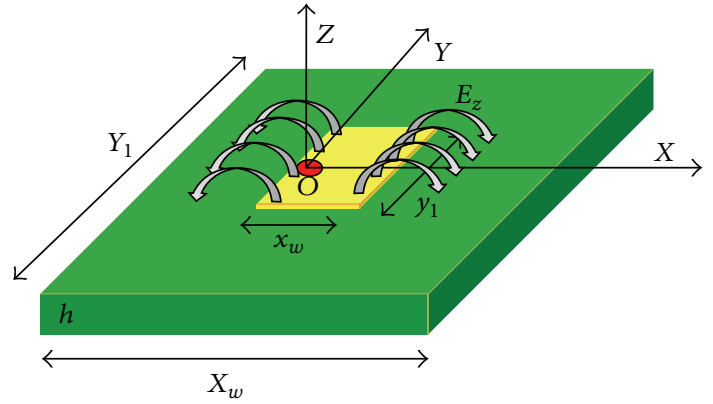

FIGURE 6: SRP structure.

where $c$ is the velocity of light in free space, $\varepsilon_{r}$ is the dielectric constant, and $P$ is the period of slots. If the frequency conditions (4)-(6) are satisfied, the leaky cable produces radiation waves. Otherwise, the leaky cable generates surface waves. The suitable length of $P$ and slot size will optimize the composition of radiation and surface waves for the desired applications.

Figure 6 shows the SRP structure. The substrate material is the FR4 circuit board with the dielectric constant $\varepsilon_{r}$ of 4.4 and thickness $h$ of $1 \mathrm{~mm}$. The dimensions are $X_{w}=50 \mathrm{~mm}$, $Y_{l}=50 \mathrm{~mm}, x_{w}=10 \mathrm{~mm}$, and $y_{l}=20 \mathrm{~mm}$. The signals are fed into the SRP at position $O$, as indicated in Figure 6.

The electric field as a function of the position is as follows:

$$
E_{z}=E_{o} \cos \left(\frac{\pi x}{x}\right) .
$$

The magnetic flow density on the patch of the SRP is

$$
J=-e_{n} \cdot e_{z} \cdot E_{z} .
$$

The minimum electric field required to activate the tag, $E_{r}$, is derived as follows:

$$
\begin{gathered}
E_{r}=\left[P_{r} \cdot\left(4 \cdot \frac{\pi}{\lambda^{2}}\right) \cdot\left(\frac{377}{G_{r}}\right) \cdot\left(\frac{1}{\tau}\right)\right]^{0.5}, \\
S=\frac{G_{t} P_{t}}{4 \pi R^{2}}=\frac{E^{2}}{377}, \\
\tau=\frac{4 \cdot R_{\mathrm{ic}} \cdot R_{\mathrm{ant}}}{\left|Z_{\mathrm{ant}}+Z_{\mathrm{ic}}\right|^{2}}
\end{gathered}
$$

where $P_{r}$ is the received power of the tag at a distance from the antenna of the reader, $\lambda$ is the operation wavelength, $G_{r}$ is the tag antenna gain, $S$ is the electric field obtained per unit area, $G_{t}$ is the reader antenna gain, $P_{t}$ is the output power of the reader, $R$ is the distance from reader antenna, $E$ is the electric field, $\tau$ is the power transfer efficiency from the antenna of the RFID tag to the radio-frequency integrated circuit (RFIC) of the RFID tag, $R_{\mathrm{ic}}$ is the real RFIC impedance, $R_{\mathrm{ant}}$ is the real antenna impedance, $Z_{\text {ant }}$ is the antenna impedance, and $Z_{\text {ic }}$ is the RFIC impedance. 


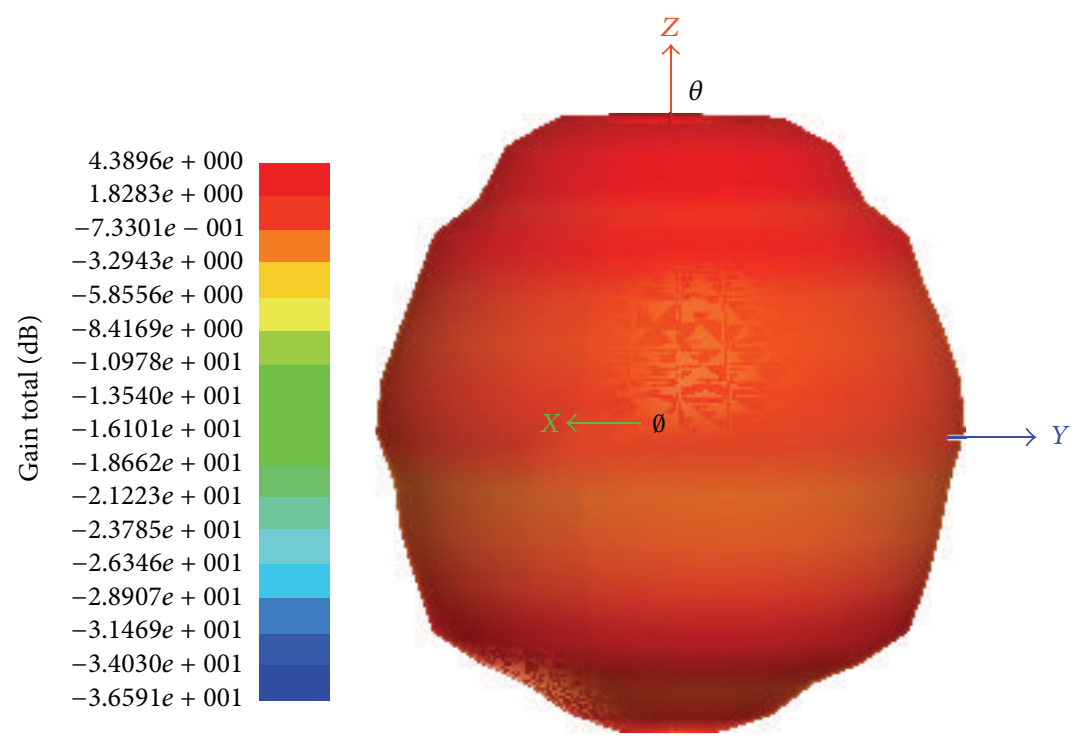

FIGURE 7: 3D electromagnetic model of ESWLCA.

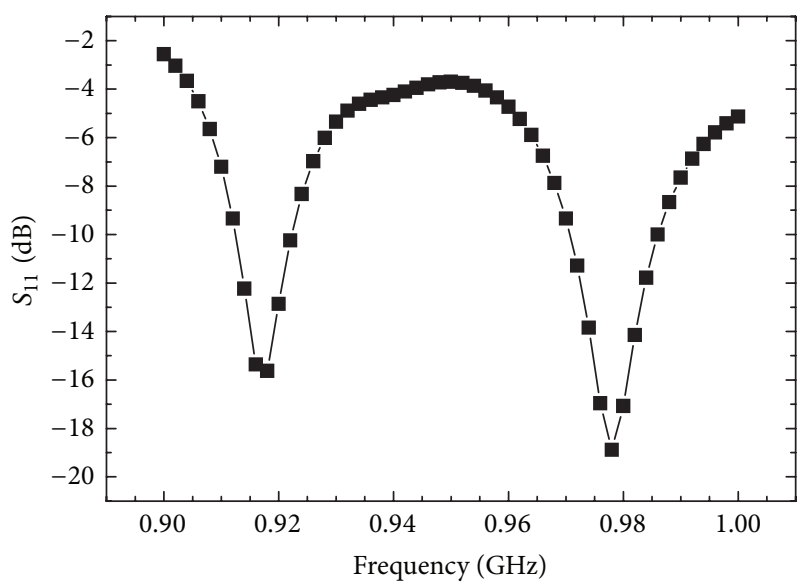

FIGURE 8: $S_{11}$ return loss characteristics of ESWLCA.

\section{System Design}

The design of the RFID sensing system is based on a fullwave electromagnetic simulator, Ansoft HFSS. The system is designed to be operated at a UHF frequency band of $860-960 \mathrm{MHz}$. The output power of the RFID reader is $30 \mathrm{dBm}$. According to (9), the minimum electric field required to activate the RFID tag is $4.8 \mathrm{~V} / \mathrm{m}$. Figure 7 shows the three-dimensional (3D) electromagnetic model of the ESWLCA. Figure 8 shows the return loss characteristics of the ESWLCA.

Figure 9 is the equivalent circuit of the Alien Higgs-3 RFIC, in which the parallel capacitance $C_{p}$ is $1.3 \mathrm{pF}$ and the

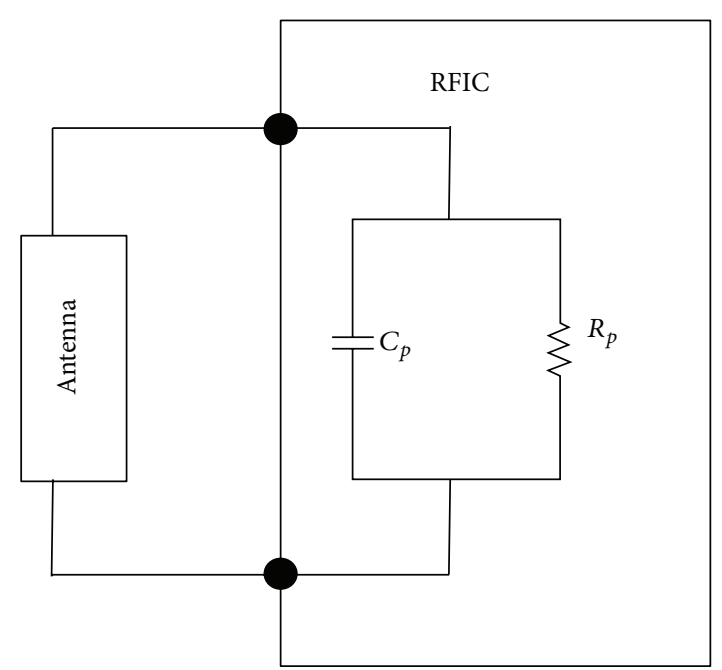

FIgURE 9: RFIC equivalent circuit.

parallel resistance $R_{p}$ is $1.5 \mathrm{kohms}$. The RFIC impedance $Z_{\mathrm{ic}}$ can be determined by

$$
Z_{\text {ic }}=\frac{R_{p}}{1+\omega^{2} C_{p}^{2} R_{p}^{2}}-\frac{j \omega C_{p} R_{p}^{2}}{1+\omega^{2} C_{p}^{2} R_{p}^{2}} .
$$

The impedance of the Higgs-3 RFIC at $920 \mathrm{MHz}$ is $31-$ j216 ohms.

The back-radiation power of the RFID tag of the battery, $P_{\text {back }}$, is

$$
P_{\text {back }}=\frac{P_{\text {th }}}{\tau} \cdot G_{r} \cdot\left|\frac{Z_{\text {ic }}-Z_{\text {ant }}}{Z_{\text {ic }}+Z_{\text {ant }}}\right|^{2},
$$

where $P_{\text {th }}$ is the threshold power to activate the RFIC. 


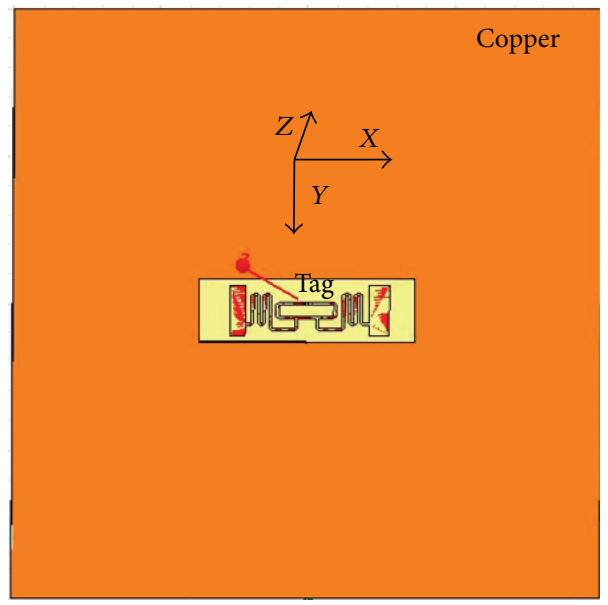

FIGURE 10: Environment of RFID tag of battery.

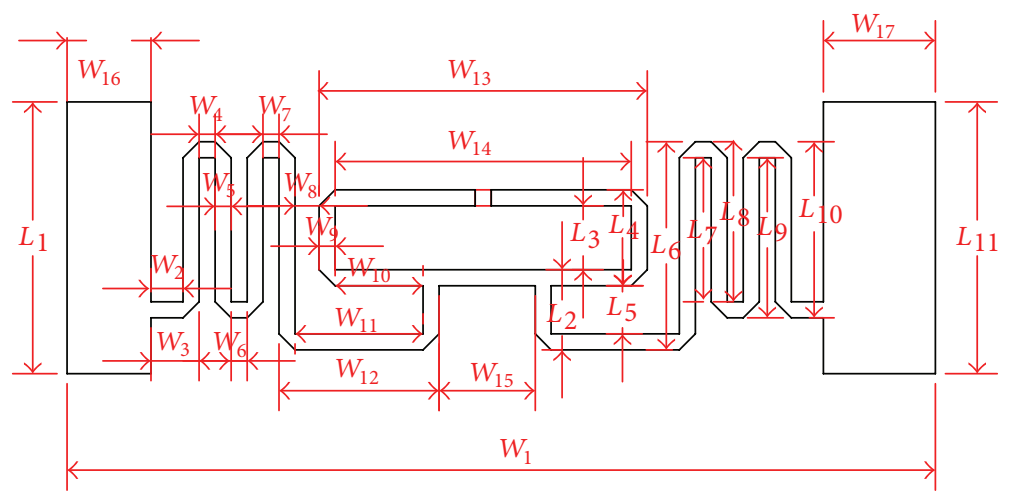

Figure 11: Design diagram of RFID tag.

The radar cross-section of the antenna of the tag, $\sigma$, is

$$
\begin{aligned}
\sigma= & \frac{P_{\text {back }}}{\left(P_{t} \cdot G_{t}\right) / 4 \pi R^{2}} \\
= & \left(\left(\left(\left(P_{t} \cdot G_{t}\right) / 4 \pi R^{2}\right) \cdot \lambda / 4 \pi \cdot G_{r}\right)\right. \\
& \left.\quad \cdot G_{r} \cdot\left|\left(Z_{\text {ic }}-Z_{\text {ant }}\right) /\left(Z_{\text {ic }}+Z_{\text {ant }}\right)\right|^{2}\right) \\
& \times\left(\left(P_{t} \cdot G_{t}\right) / 4 \pi R^{2}\right)^{-1} .
\end{aligned}
$$

\section{Results and Discussion}

Figure 10 shows the environment of the RFID tag of the battery. A copper metal object with the size of $200 \times 200 \times$ $1 \mathrm{~mm}^{3}$ is $2 \mathrm{~mm}$ below the RFID tag.

Figure 11 shows the design diagram of the RFID tag with the dimensions of $L_{1}=17 \mathrm{~mm}, L_{2}=5 \mathrm{~mm}, L_{3}=4 \mathrm{~mm}, L_{4}=$ $6 \mathrm{~mm}, L_{5}=3 \mathrm{~mm}, L_{6}=13 \mathrm{~mm}, L_{7}=9 \mathrm{~mm}, L_{8}=$ $10 \mathrm{~mm}, L_{9}=10 \mathrm{~mm}, L_{10}=11 \mathrm{~mm}, L_{11}=17 \mathrm{~mm}, W_{1}=$ $54.25 \mathrm{~mm}, W_{2}=2 \mathrm{~mm}, W_{3}=3 \mathrm{~mm}, W_{4}=1 \mathrm{~mm}, W_{5}=$ $1 \mathrm{~mm}, W_{6}=1 \mathrm{~mm}, W_{7}=1 \mathrm{~mm}, W_{8}=1.5 \mathrm{~mm}, W_{9}=1 \mathrm{~mm}$, $W_{10}=5.5 \mathrm{~mm}, W_{11}=8 \mathrm{~mm}, W_{12}=10 \mathrm{~mm}, W_{13}=20.5 \mathrm{~mm}$, $W_{14}=18.5 \mathrm{~mm}, W_{15}=6 \mathrm{~mm}, W_{16}=5.25 \mathrm{~mm}$, and $W_{17}=$ $7 \mathrm{~mm}$.

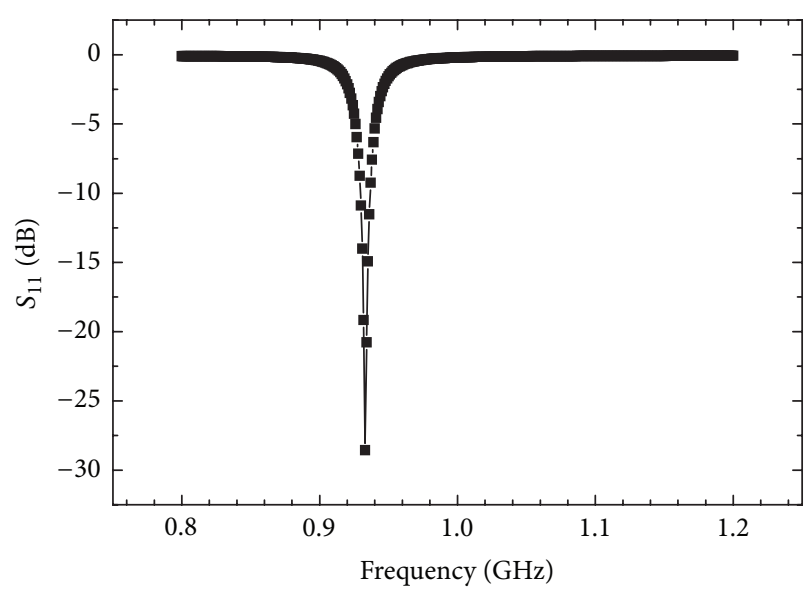

FIgURE 12: $S_{11}$ return loss characteristics of RFID tag of battery.

Figure 12 shows the $S_{11}$ return loss characteristics of the RFID tag of the battery. The tag is suitable for objects with metal surfaces. The $S_{11}$ is $-28.6 \mathrm{~dB}$ at $930 \mathrm{MHz}$. The antenna impedance is $Z_{\text {ant }}=19+j 186$ ohms. Figure 13 shows the $3 \mathrm{D}$ pattern of the RFID tag of the battery. The Alien Higgs- 3 turn on power sensitivity is $-18 \mathrm{dBm}$. 


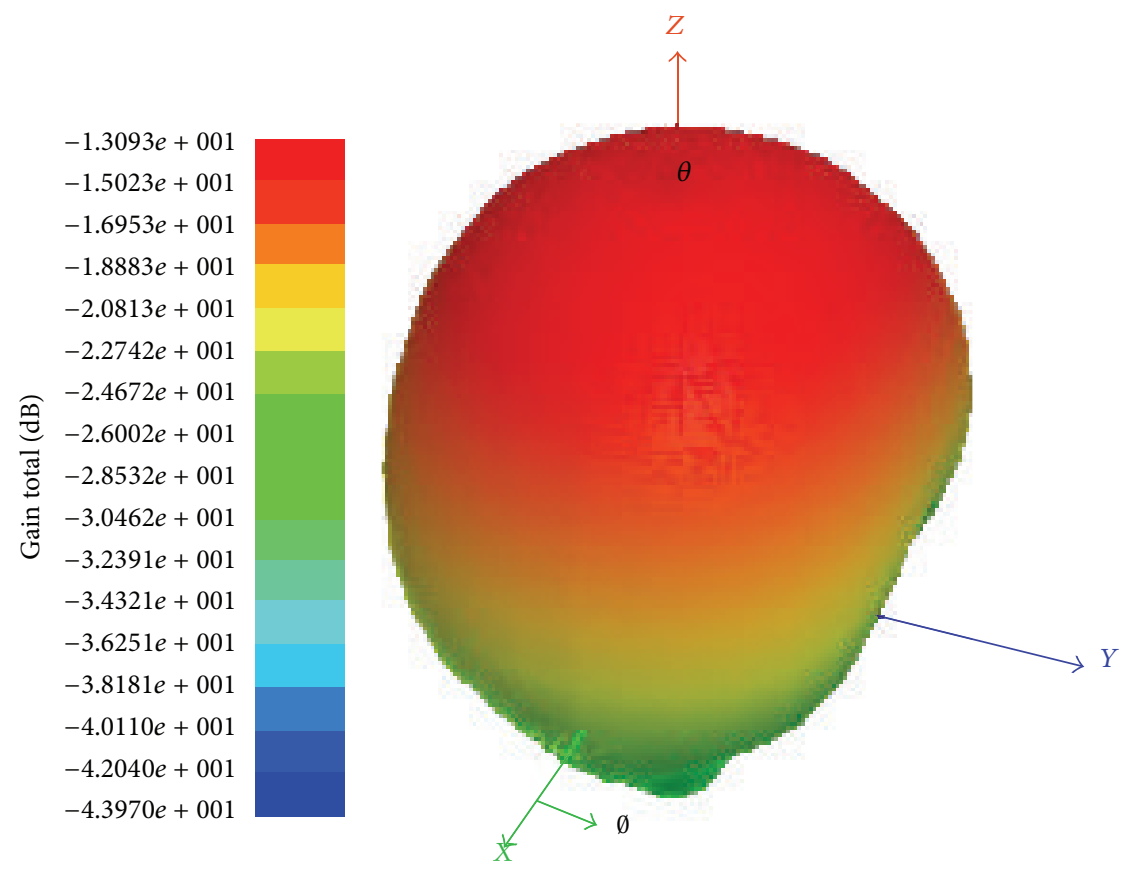

FIGURE 13: 3D pattern of RFID tag of battery.

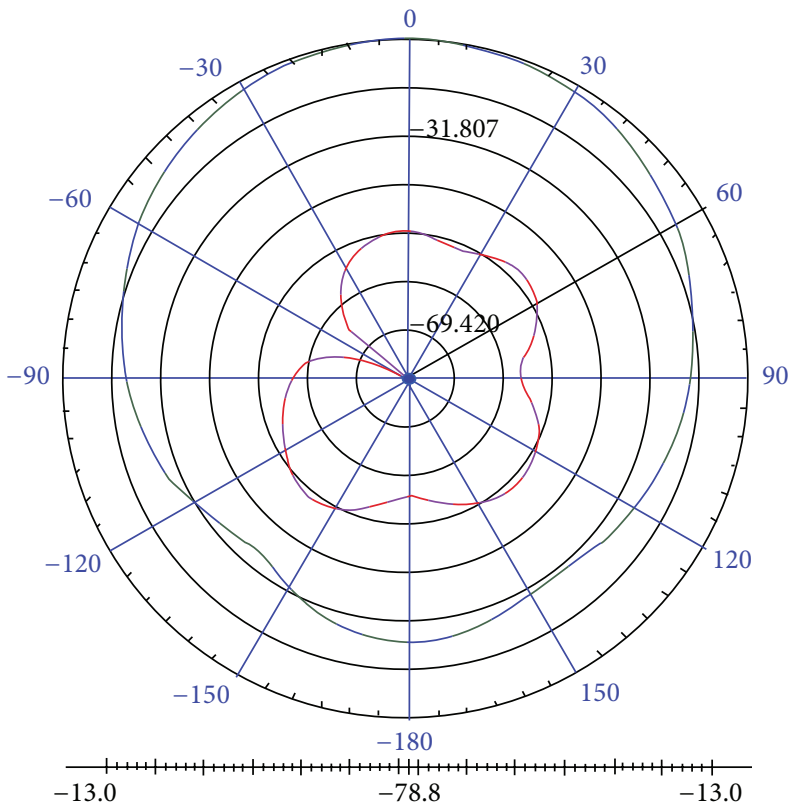

FIGURE 14: 2D pattern of RFID tag of battery.

Figure 14 shows the two-dimensional (2D) pattern of the RFID tag of the battery. The maximum gain of the antenna of the RFID tag of the battery $G_{r}$ is $-13 \mathrm{dBm}$. According to (11), the power transfer efficiency from the antenna of the RFID tag of the battery to the Higgs-3 RFIC is $\tau=0.6$.

The surface electric field distribution of the ESWLCA is analyzed as the RFID reader feeds $30 \mathrm{dBm}$ output power into the ESWLCA. Figure 15 shows the surface electric field distribution of the ESWLCA at the location of $1 \mathrm{~mm}$ from the ESWLCA. The maximum electric field $E_{\max }$ is greater than $100 \mathrm{~V} / \mathrm{m}$ and the minimum electric field $E_{\min }$ is $80 \mathrm{~V} / \mathrm{m}$. The RFID tag requires the minimum electric field of $4.8 \mathrm{~V} / \mathrm{m}$ for operation.

Figures 16, 17, and 18 illustrate the electric field distribution at a distance $H$ from the ESWLCA. Figure 16 shows the 


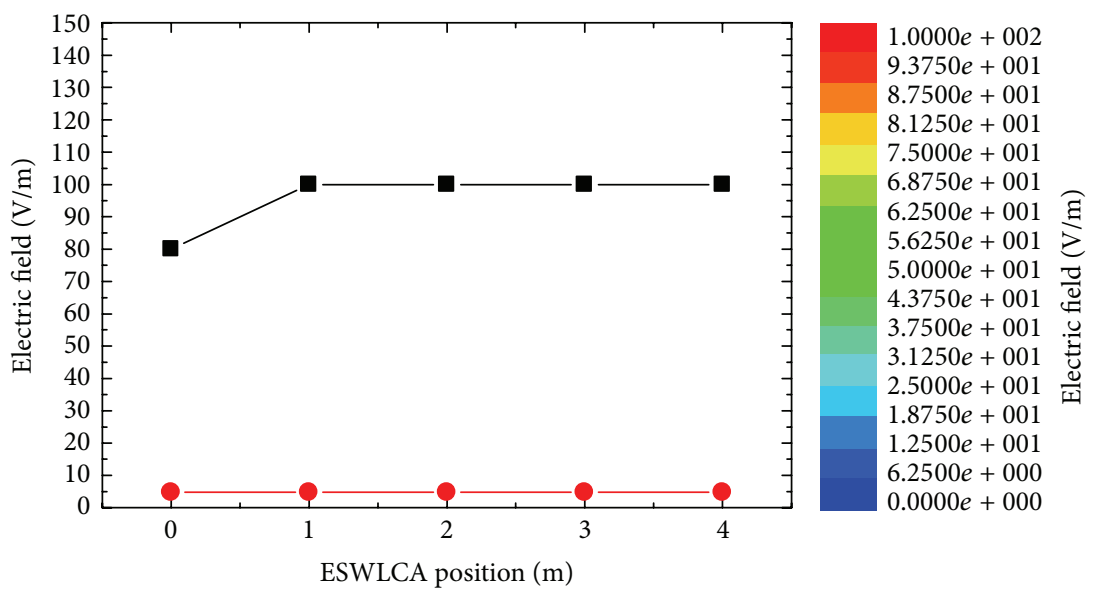

- Surface electric field

$\multimap E_{r}=4.8 \mathrm{~V} / \mathrm{m}$

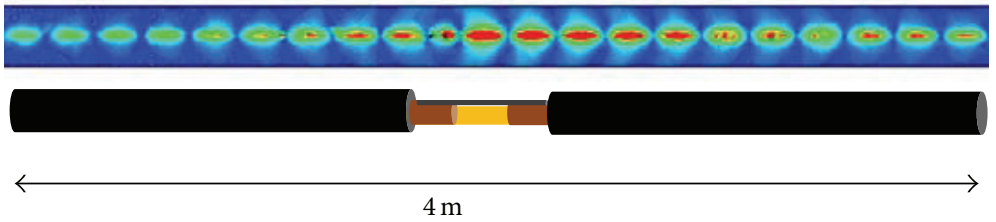

FIGURE 15: Surface electric field distribution of ESWLCA.

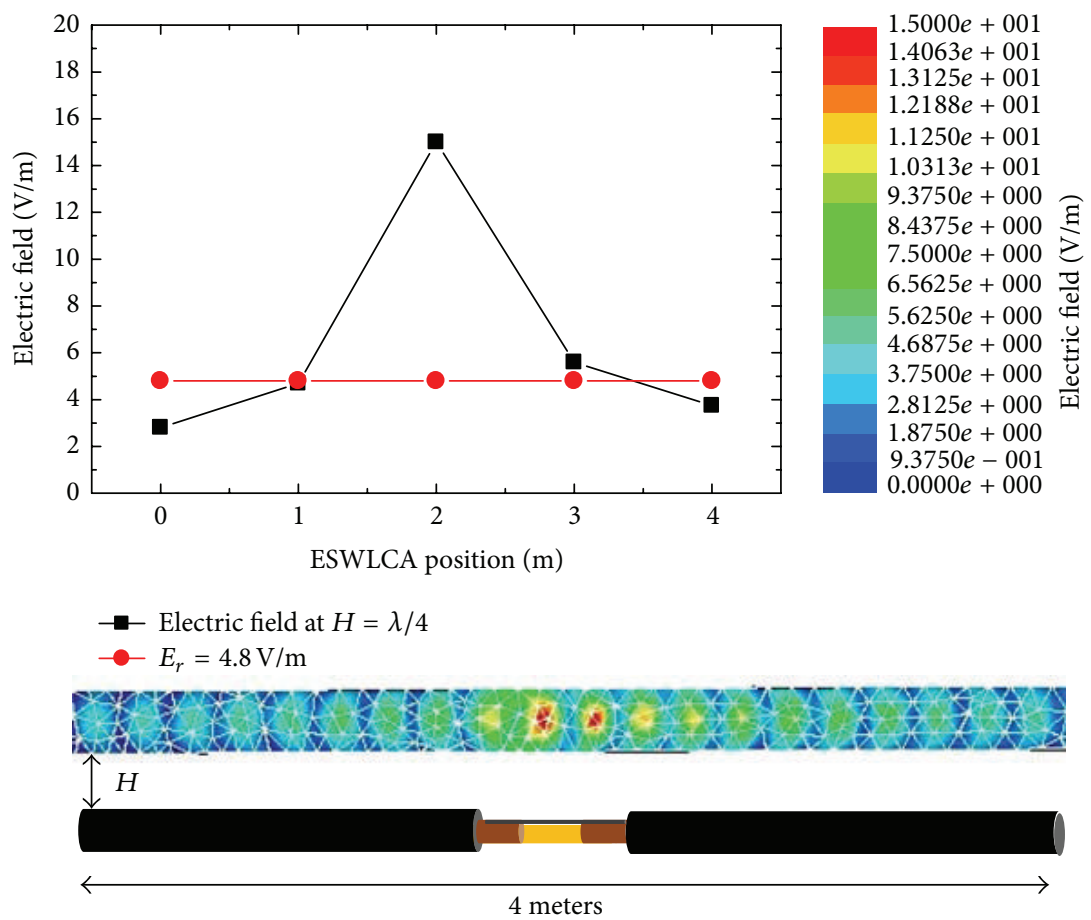

FIGURE 16: Electric field distribution of ESWLCA at $H=\lambda / 4$. 


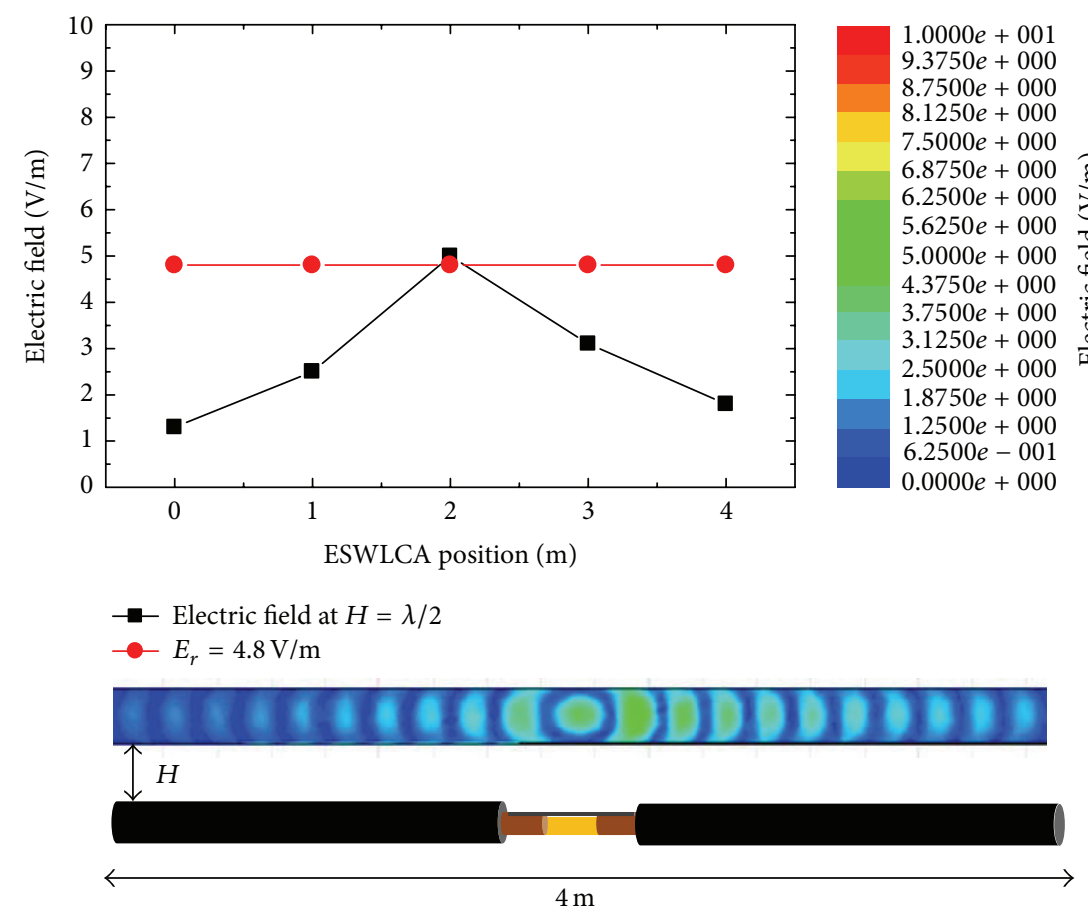

FIgURE 17: Electric field distribution of ESWLCA at $H=\lambda / 2$.

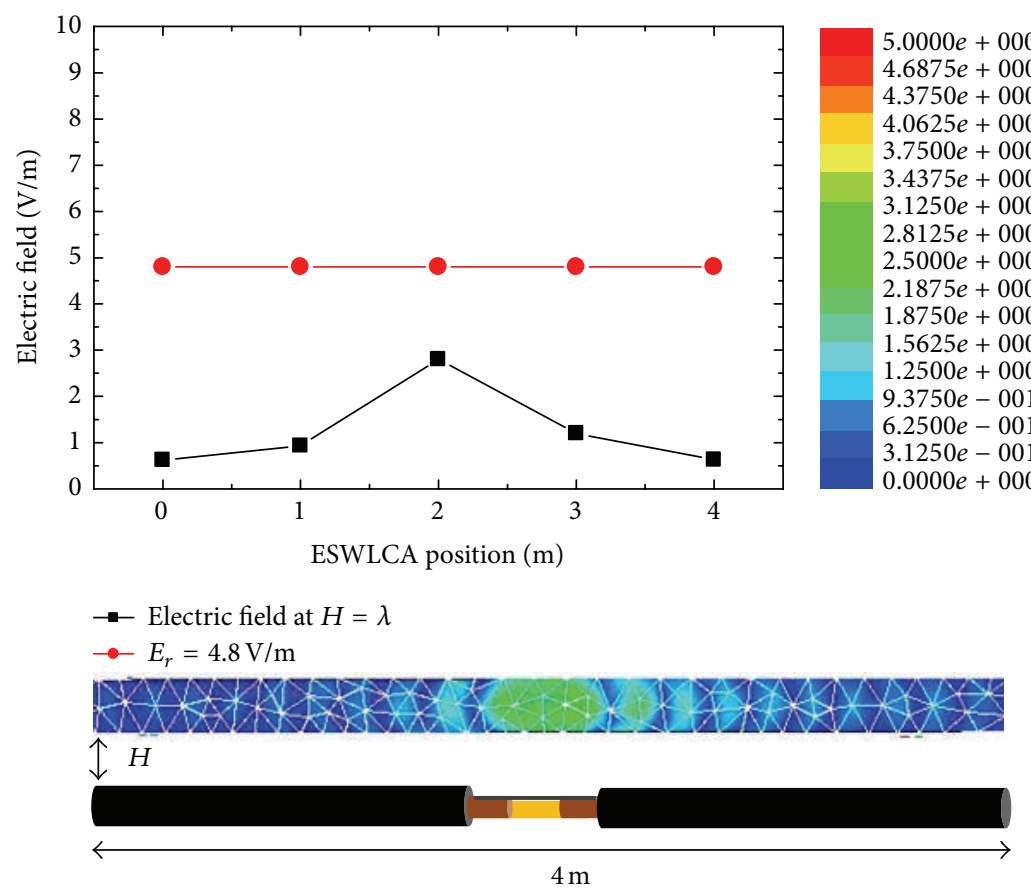

FIGURE 18: Electric field distribution of ESWLCA at $H=\lambda$.

electric field distribution of the ESWLCA at a quarter of a wavelength from the ESWLCA $(H=\lambda / 4)$. The maximum electric field $E_{\max }$ is $15 \mathrm{~V} / \mathrm{m}$ and the minimum electric field $E_{\min }$ is $2.8 \mathrm{~V} / \mathrm{m}$.
Figure 17 shows the electric field distribution of the ESWLCA at half a wavelength from the ESWLCA $(H=\lambda / 2)$. The maximum electric field $E_{\max }$ is $5 \mathrm{~V} / \mathrm{m}$ and the minimum electric field $E_{\min }$ is $1.3 \mathrm{~V} / \mathrm{m}$. 


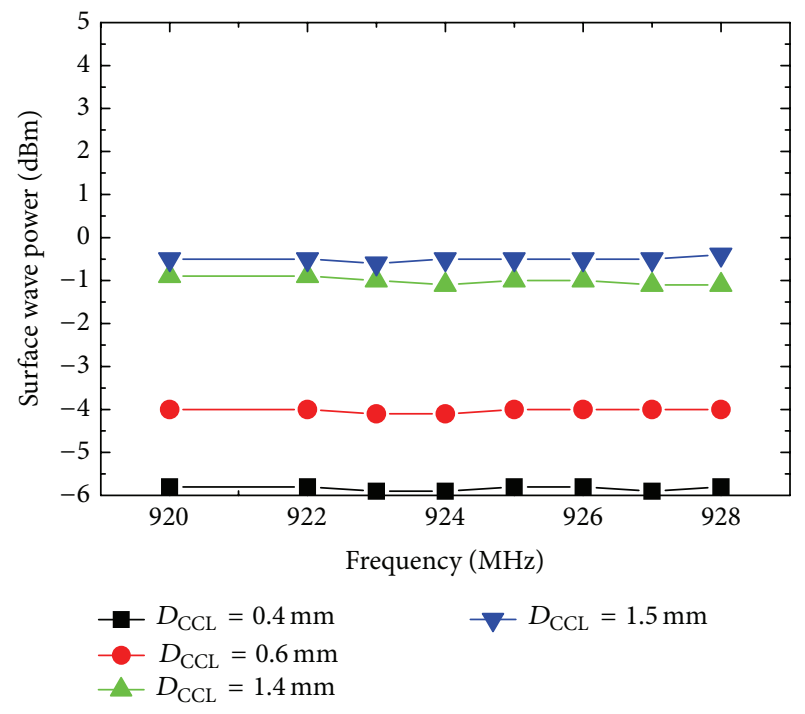

FIGURE 19: Measurement characteristics of surface wave power.

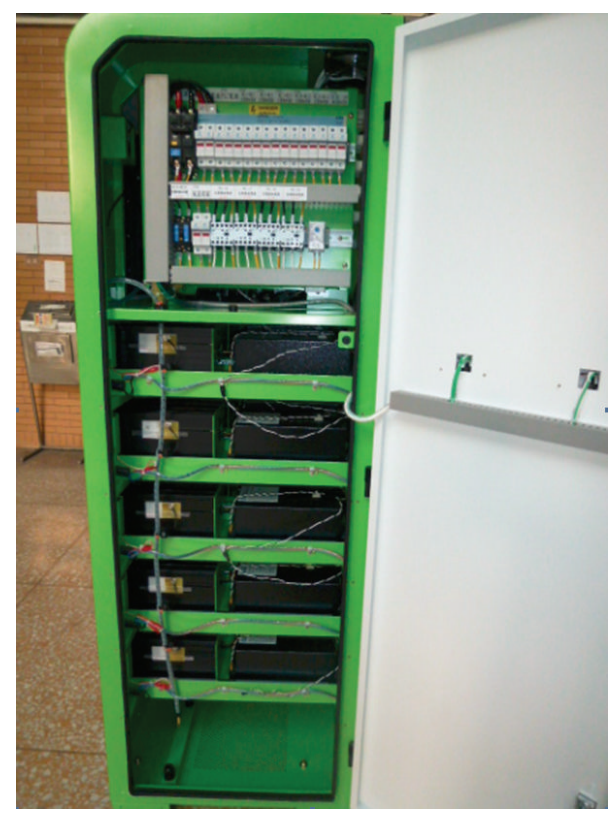

FIGURE 20: Completed RFID sensing system for BES.

Figure 18 shows the electric field distribution of the ESWLCA at a full wavelength from the ESWLCA $(H=\lambda)$. The maximum electric field $E_{\max }$ is $2.8 \mathrm{~V} / \mathrm{m}$ and the minimum electric field $E_{\min }$ is $0.62 \mathrm{~V} / \mathrm{m}$.

Figure 19 shows the measurement characteristics of the surface wave power as a function of the diameter of the CCL $\left(D_{\mathrm{CCL}}\right)$ at a source power of $0 \mathrm{dBm}$. The surface wave power increases with the increasing $D_{\mathrm{CCL}}$.

Figure 20 shows the completed sensing system for the BES. The ESWLCA is suitable for the BES which is a small space in a metallic cabinet. The leaky wave is radiated from the open slot in the transmission line of the ESWLCA. The electromagnetic wave propagates along the surface of the transmission line and transmits to the SRP through the bendable CCL. The CCL can still easily transmit the electromagnetic wave even in a closed metallic environment where the barcode approach is not applicable.

\section{Conclusion}

The state-of-the-art UHF RFID sensing system for the BES of electric motorcycles has been developed. The ESWLCA, CCL, and SRP are designed to overcome the metallic environment in a BES cabinet. The RFID sensing system demonstrates excellent characteristics and shows great potential for the modern BES of electric vehicles.

\section{Conflict of Interests}

The authors declare that there is no conflict of interests regarding the publication of this paper.

\section{Acknowledgment}

This work was supported in part by the Ministry of Science and Technology of Taiwan, R.O.C. under Contracts NSC 1012218-E-018-001, NSC 102-2218-E-018-002, and MOST 1032221-E-018-021.

\section{References}

[1] P. Mirchandani, J. Adler, and O. B. G. Madsen, "New logistical issues in using electric vehicle fleets with battery exchange infrastructure," Procedia-Social and Behavioral Sciences, vol. 108, pp. 3-14, 2014.

[2] Y.-W. Wang, "Locating battery exchange stations to serve tourism transport: a note," Transportation Research D: Transport and Environment, vol. 13, no. 3, pp. 193-197, 2008.

[3] Y.-L. Lai and C.-C. Chen, "An intelligent RFID fall notification system," International Journal of Innovative Computing, Information and Control, vol. 7, no. 6, pp. 3133-3145, 2011.

[4] C.-S. Cheng, H. H. Chang, Y.-T. Chen et al., "Accurate location tracking based on active RFID for health and safety monitoring," in Proceedings of the 3rd International Conference on Bioinformatics and Biomedical Engineering (iCBBE '09), pp. 1-4, Beijing, China, June 2009.

[5] C. W. Schneider, J. Tautz, B. Grünewald, and S. Fuchs, "RFID tracking of sublethal effects of two neonicotinoid insecticides on the foraging behavior of Apis mellifera," PLoS ONE, vol. 7, no. 1, Article ID e30023, pp. 1-9, 2012.

[6] C.-H. Chang, Y.-L. Lai, and C.-C. Chen, "Implement the RFID position based system of automatic tablets packaging machine for patient safety," Journal of Medical Systems, vol. 36, no. 6, pp. 3463-3471, 2012.

[7] S. F. Wamba and E. W. T. Ngai, "Importance of the relative advantage of RFID as enabler of asset management in the healthcare: results from a Delphi study," in Proceedings of the 45th Hawaii International Conference on System Sciences (HICSS '12), pp. 2879-2889, Maui, Hawaii, USA, January 2012.

[8] Y.-L. Lai and J. Cheng, "A 2.45-GHz RFID wireless-sensornetwork location tracking system," in Proceedings of the IEEE 17th International Symposium on Consumer Electronics (ISCE '13), pp. 133-134, Hsinchu, Taiwan, June 2013. 
[9] H. Jabbar, T. Jeong, J. Hwang, and G. Park, "Viewer identification and authentication in IPTV using RFID technique," IEEE Transactions on Consumer Electronics, vol. 54, no. 1, pp. 105-109, 2008.

[10] C.-L. Chen, Y.-L. Lai, C.-C. Chen, Y.-Y. Deng, and Y.-C. Hwang, "RFID ownership transfer authorization systems conforming EPCglobal class-1 generation-2 standards," International Journal of Network Security, vol. 13, no. 1, pp. 41-48, 2011.

[11] Y.-L. Lai and J. Cheng, "A cloud-storage RFID location tracking system," IEEE Transactions on Magnetics, vol. 50, no. 7, Article ID 3501004, 2014.

[12] N. Raza, V. Bradshaw, and M. Hague, "Applications of RFID technology," in Proceedings of the IEE Colloquium on RFID Technology, pp. 1/1-1/5, London, UK, October 1999.

[13] G. Steiner, H. Zangl, P. Fulmek, and G. Brasseur, "A tuning transformer for the automatic adjustment of resonant loop antennas in RFID systems," in Proceedings of the IEEE International Conference on Industrial Technology (ICIT '04), vol. 2, pp. 912-916, Hammamet, Tunisia, December 2004.

[14] C. C. Hennon, C. N. Helms, K. R. Knapp, and A. R. Bowen, "An objective algorithm for detecting and tracking tropical cloud clusters: implications for tropical cyclogenesis prediction," Journal of Atmospheric and Oceanic Technology, vol. 28, no. 8, pp. 1007-1018, 2011.

[15] I.-C. Lin, C.-W. Yang, and S.-C. Tsaur, "Nonidentifiable RFID privacy protection with ownership transfer," International Journal of Innovative Computing, Information and Control, vol. 6, no. 5, pp. 2341-2351, 2010.

[16] M. Buckner, R. Crutcher, M. R. Moore, and B. Whitus, "MICLOG RFID tag program enables total asset visibility," in Proceedings of the Military Communications Conference (MILCOM '02), vol. 2, pp. 1422-1426, Anaheim, Calif, USA, October 2002.

[17] W. D. Yu, P. Ray, and T. Motoc, "A RFID technology based wireless mobile multimedia system in healthcare," in Proceedings of the 8th International Conference on e-Health Networking, Applications and Services (HEALTHCOM '06), pp. 1-8, New Delhi, India, August 2006.

[18] D.-G. Yun, J.-M. Lee, M.-J. Yu, and S.-G. Choi, “Agent-based user mobility support mechanism in RFID networking environment," IEEE Transactions on Consumer Electronics, vol. 55, no. 2, pp. 800-804, 2009.

[19] Y.-S. Yen, F. Lin, and H.-C. Chao, "Integrated residential gateway: easy IA management with P2P community using RFID," IEEE Transactions on Consumer Electronics, vol. 51, no. 3, pp. 824-830, 2005.

[20] R. Tesoriero, J. A. Gallud, M. D. Lozano, and V. M. R. Penichet, "Tracking autonomous entities using RFID technology," IEEE Transactions on Consumer Electronics, vol. 55, no. 2, pp. 650655, 2009.

[21] R. Tesoriero, J. A. Gallud, M. Lozano, and V. M. R. Penichet, "Using active and passive RFID technology to support indoor location-aware systems," IEEE Transactions on Consumer Electronics, vol. 54, no. 2, pp. 578-583, 2008.

[22] S. S. Manapure, H. Darabi, V. Patel, and P. Banerjee, "A comparative study of radio frequency-based indoor location sensing systems," in Proceedings of the IEEE International Conference on Networking, Sensing and Control, vol. 2, pp. 1265-1270, Taipei, Taiwan, March 2004.
[23] H. Hontani, M. Nakagawa, T. Kugimiya, K. Baba, and M. Sato, "A visual tracking system using an RFID-tag", in Proceedings of the SICE Annual Conference, pp. 2720-2723, Sapporo, Japan, August 2004.

[24] T. Cao and P. Shen, "Cryptanalysis of two RFID authentication protocols," International Journal of Network Security, vol. 9, no. 1, pp. 95-100, 2009.

[25] S. L. Garfinkel, A. Juels, and R. Pappu, "RFID privacy: an overview of problems and proposed solutions," IEEE Security and Privacy, vol. 3, no. 3, pp. 34-43, 2005.

[26] J. M. Sieglaff, D. C. Hartung, W. F. Feltz, L. M. Cronce, and V. Lakshmanan, "A satellite-based convective cloud object tracking and multipurpose data fusion tool with application to developing convection," Journal of Atmospheric and Oceanic Technology, vol. 30, no. 3, pp. 510-525, 2013.

[27] S. Han, H. Lim, and J. Lee, "An efficient localization scheme for a differential-driving mobile robot based on RFID system," IEEE Transactions on Industrial Electronics, vol. 54, no. 6, pp. $3362-$ 3369, 2007.

[28] H. Lei and T. Cao, "RFID protocol enabling ownership transfer to protect against traceability and DoS attacks," in Proceedings of the 1st International Symposium on Data, Privacy and ECommerce, pp. 508-510, Chengdu, China, November 2007.

[29] J.-C. Lu, Y.-Y. Chen, J.-M. Wang, J.-K. Jan, C.-C. Chen, and Y.L. Lai, "Study and implementation of RFID eseals for power meters," in Proceedings of the 2nd International Conference on Innovations in Bio-Inspired Computing and Applications (IBICA '11), pp. 352-355, Shenzhan, China, December 2011.

[30] B. Toiruul and K. Lee, "An advanced mutual-authentication algorithm using AES for RFID systems," IJCSNS International Journal of Computer Science and Network Security, vol. 6, no. 9B, pp. 156-162, 2006.

[31] D. Henrici and P. Müller, "Hash-based enhancement of location privacy for radio-frequency identification devices using varying identifiers," in Proceedings of the 2nd IEEE Annual Conference on Pervasive Computing and Communications Workshops (PERCOMW'04), pp. 149-153, Orlando, Fla, USA, March 2004.

[32] A. Juels, "Strengthening EPC tags against cloning," in Proceedings of the 4th ACM Workshop on Wireless Security (WiSe '05), pp. 67-76, Cologne, Germany, September 2005.

[33] K. H. S. S. Koralalage, S. M. Reza, J. Miura, Y. Goto, and J. Cheng, "POP method: an approach to enhance the security and privacy of RFID systems used in product lifecycle with an anonymous ownership transferring mechanism," in Proceedings of the ACM Symposium on Applied Computing (SAC '07), pp. 270-275, Seoul, Republic of Korea, March 2007.

[34] D. Molnar, A. Soppera, and D. Wagner, "A scalable, delegatable pseudonym protocol enabling ownership transfer of RFID tags," in Selected Areas in Cryptography: 12th International Workshop, SAC 2005, Kingston, ON, Canada, August 2005, vol. 3897 of Lecture Notes in Computer Science, pp. 276-290, 2006.

[35] K. Osaka, T. Takagi, K. Yamazaki, and O. Takahashi, "An efficient and secure RFID security method with ownership transfer," in Proceedings of the International Conference on Computational Intelligence and Security (CIS '06), pp. 1090-1095, Guangzhou, China, November 2006.

[36] J. Saito, J.-C. Ryou, and K. Sakurai, "Enhancing privacy of universal re-encryption scheme for RFID tags," in Embedded and Ubiquitous Computing: International Conference EUC 2004, Aizu-Wakamatsu City, Japan, August 2004, vol. 3207 of Lecture Notes in Computer Science, pp. 879-890, 2004. 
[37] Y. Seo, T. Asano, H. Lee, and K. Kim, "A lightweight protocol enabling ownership transfer and granular data access of RFID tags," in Proceedings of the Symposium on Cryptography and Information Security (SCIS '07), pp. 1-6, Sasebo, Japan, January 2007.

[38] S. A. Weis, S. E. Sarma, R. L. Rivest, and D. W. Engels, "Security and privacy aspects of low-cost radio frequency identification systems," in Security in Pervasive Computing: 1st International Conference SPC 2003, Boppard, Germany, March 2003, vol. 2802 of Lecture Notes in Computer Science, pp. 201-212, 2004.

[39] Y. P. Zhang, "Indoor radiated-mode leaky feeder propagation at 2.0 GHz," IEEE Transactions on Vehicular Technology, vol. 50, no. 2, pp. 536-545, 2001.

[40] J. H. Wang and K. K. Mei, "Theory and analysis of leaky coaxial cables with periodic slots," IEEE Transactions on Antennas and Propagation, vol. 49, no. 12, pp. 1723-1732, 2001.

[41] P. R. Foster and R. A. Burberry, "Antenna problems in RFID systems," in Proceedings of the IEE Colloquium on RFID Technology, pp. 3/1-3/5, London, UK, October 1999.

[42] S.-T. Kim, G.-H. Yun, and H.-K. Park, "Numerical analysis of the propagation characteristics of multiangle multislot coaxial cable using moment method," IEEE Transactions on Microwave Theory and Techniques, vol. 46, no. 3, pp. 269-279, 1998.

[43] J. R. Wait, "Electromagnetic field analysis for a coaxial cable with periodic slots," IEEE Transactions on Electromagnetic Compatibility, vol. EMC-19, no. 1, pp. 7-13, 1977.

[44] J. Huang, T. Wei, S. Wu, X. Lan, J. Fan, and H. Xiao, "Coaxial cable bragg grating sensors for structural health monitoring," International Journal of Pavement Research and Technology, vol. 5, no. 5, pp. 338-342, 2012.

[45] T. Kawanishi and M. Izutsu, "Coaxial periodic optical waveguide," Optics Express, vol. 7, no. 1, pp. 10-22, 2000.

[46] K. Inomata, Y. Yamaguchi, H. Yamada, W. Tsujita, M. Shikai, and K. Sumi, "Accuracy of 2-dimensional object location estimation using leaky coaxial cables," IEEE Transactions on Antennas and Propagation, vol. 59, no. 6, pp. 2396-2403, 2011. 


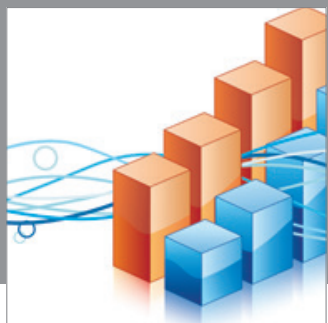

Advances in

Operations Research

mansans

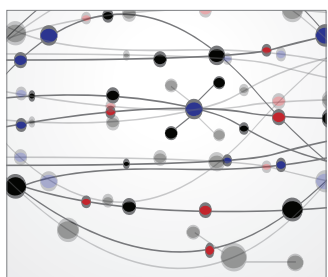

The Scientific World Journal
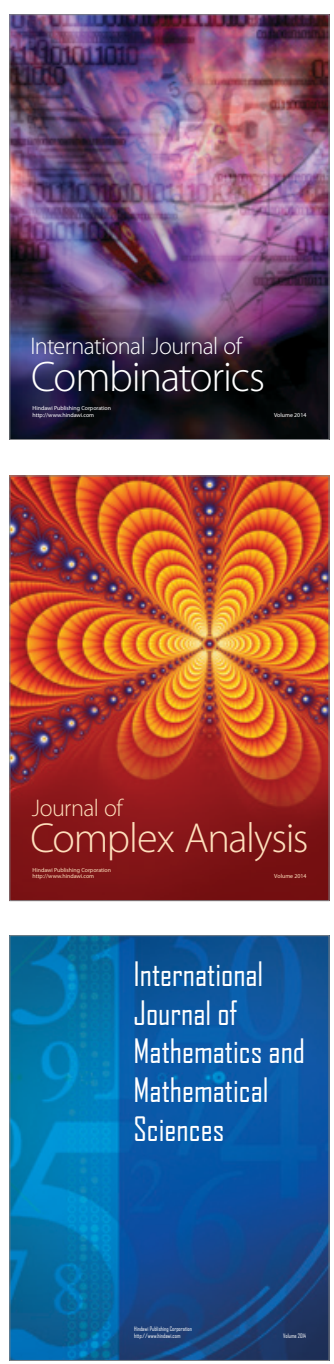
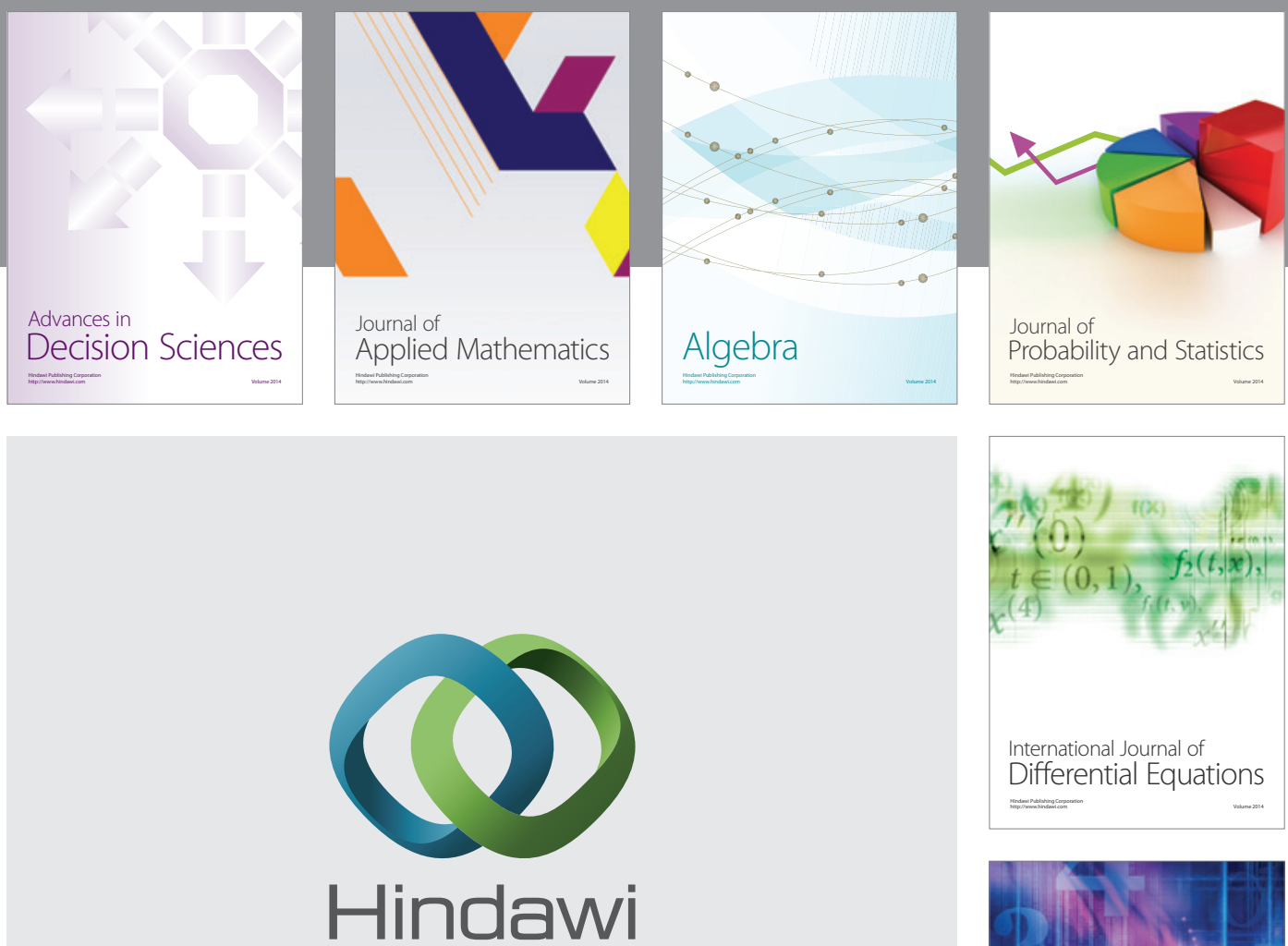

Submit your manuscripts at http://www.hindawi.com
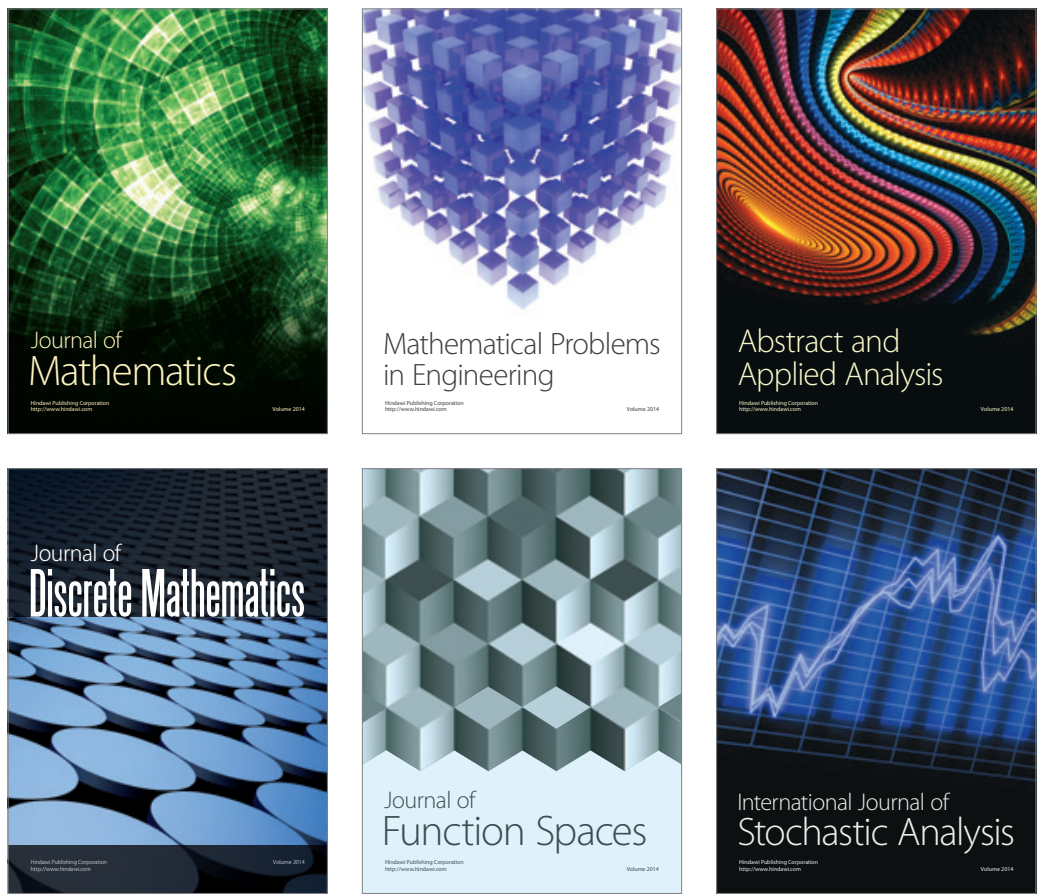

Journal of

Function Spaces

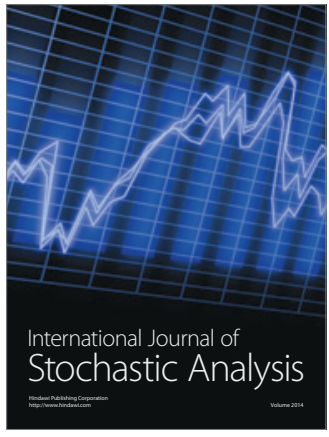

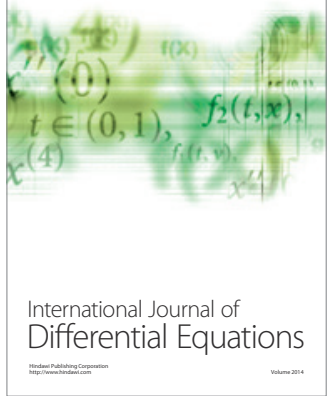
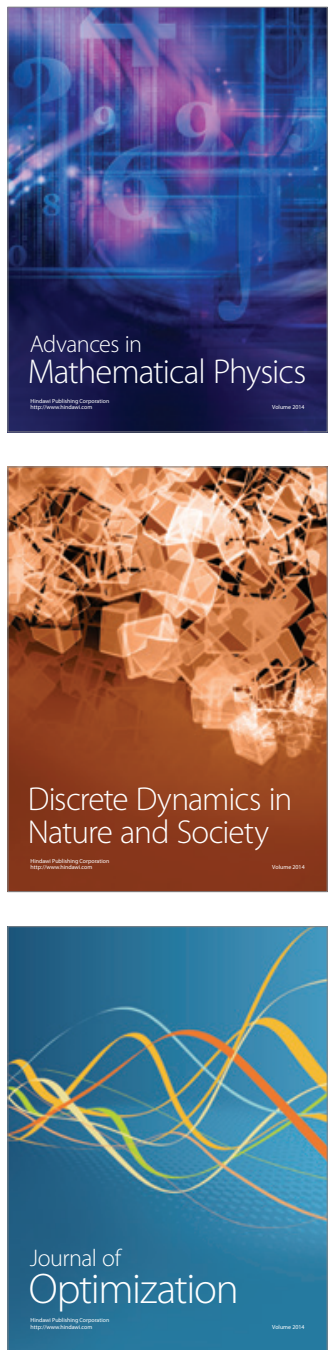